\title{
Stress on spur gear and simulation for micro hybrid systems by Ansys workbench
}

Anisul ISLAM, Md. Mashrur ISLAM

DOI: 10.30464/jmee.2019.3.1.25

Cite this article as:

Islam A., Islam M. M. Stress on spur gear and simulation for micro hybrid systems by Ansys workbench. Journal of Mechanical and Energy Engineering, Vol. 3(43), No. 1, 2019, pp. 25-30.

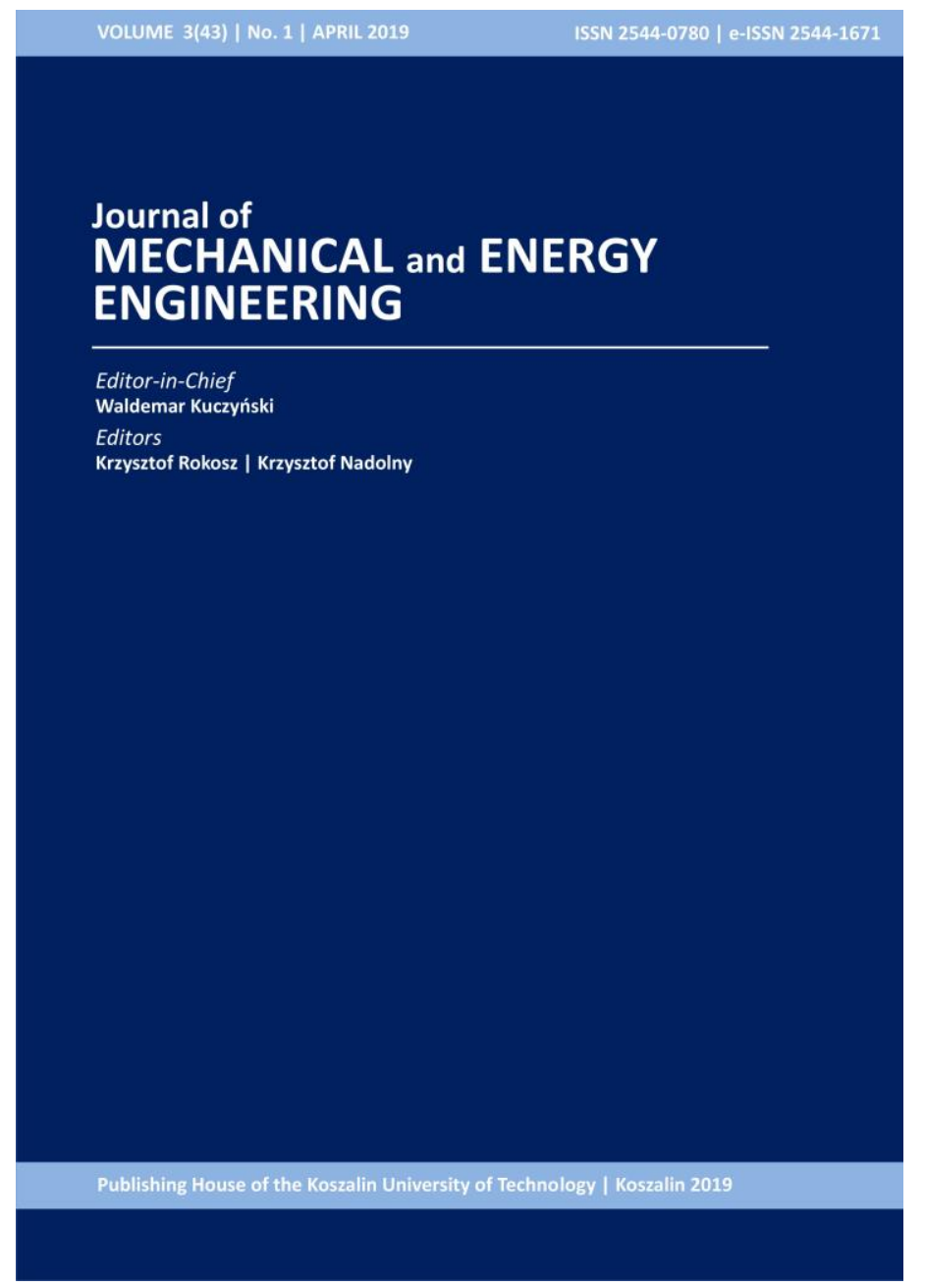

Journal of Mechanical and Energy
Engineering

Website: jmee.tu.koszalin.pl

ISSN (Print): 2544-0780

ISSN (Online): 2544-1671

Volume: 3(43)

Number: 1

Year: 2019

Pages: 25-30

\section{Article Info:}

Received 16 January 2019

Accepted 18 February 2019

\section{Open Access}

This article is distributed under the terms of the Creative Commons Attribution 4.0 (CC BY 4.0) International License (http://creativecommons.org/licenses/by/4.0/), which permits unrestricted use, distribution, and reproduction in any medium, provided you give appropriate credit to the original author(s) and the source, provide a link to the Creative Commons license, and indicate if changes were made. 


\title{
STRESS ON SPUR GEAR AND SIMULATION FOR MICRO HYBRID SYSTEMS BY ANSYS WORKBENCH
}

\author{
Anisul ISLAM ${ }^{1 *}$, Md. Mashrur ISLAM ${ }^{2}$ \\ 1* Department of Mechanical Engineering, RUET, Bangladesh, e-mail: anisulme11@ gmail.com \\ ${ }^{2}$ Department of Electrical \& Electronic Engineering, RUET, Bangladesh
}

(Received 16 January 2019, Accepted 18 February 2019)

\begin{abstract}
Spur gears are the most well-known kind of gears used in hybrid vehicle's power transmission. They have straight teeth, and are mounted on parallel shafts. In some cases, many spur gears are utilized without a moment's delay to make huge rigging decreases. In this paper how stress creates on a spur equip under various conditions and conditions and reenactments of a rigging system (two spur gears) is assessed by Ansys workbench. For this static structural and dynamic analysis modeling is utilized. A couple of spurs equip tooth in real life is by and large subjected to two sorts of cyclic stress: contact stress and twisting stress including bowing fatigue. The two stresses may not accomplish their greatest esteems at a similar purpose of contact fatigue. These sorts of failure can be limited by analysis of the issue amid the outline organize and making appropriate tooth surface profile with legitimate assembling strategies.
\end{abstract}

Keywords: Ansys workbench; Design analysis; Spur gear; Simulation; Micro Hybrid Energy

\section{INTRODUCTION}

A gear or cogwheel is a turning machine part having cut teeth, or gear-teeth, which work with another toothed part to transmit torque [1]. Geared devices can change the speed, torque, and course of a power source. Gears quite often deliver an adjustment in torque, making a mechanical preferred standpoint, through their gear proportion, and hence might be considered as a straightforward machine. The teeth of the two gears have exactly the same profile [1]. When, at least two meshing gears working with alignment known as gear train or transmission. A gear can work with a direct toothed part, called a rack, in this manner creating translation instead of rotation.

Spur gears or straight-cut gears are the least complex kind of gear. They comprise of a barrel or plate with teeth anticipating radially. In spite of the fact that the teeth are not straight-sided (but rather ordinarily of exceptional outline to accomplish a consistent drive proportion, essentially involute however less regularly cycloidal), the periphery of every tooth is straight and attuned parallel to the hub of pivot. These gears are fitted to parallel shafts works mutually accurately [2]. The tooth loads make no hub push. Spur gears are outstanding at direct speeds however they have a tendency of being noisy at elevated speeds [3].

There are a few sorts of stresses exhibit in loaded and turning gear teeth and generally two sorts of fatigue failure in spur gear. There are two hypothetical recipes, which bargain with these two-fatigue failure systems. One is the Hertz equation, which can be utilized to figure the twisting stress $[4,10]$.

Dynamic loads on the teeth, coming about because of the operation of gears are one of the vital variables deciding the dependability and durability of the transmission. Petrusevich and M.D. Genkin,1956 [5], N.A. Kovalev,1961 [6] recommended distinctive technique for dynamic analysis. Kahraman,1997 [8] and Parker,2000 [7] utilized a model with lumped parameters in which the gearing appears as hard drives associated with the versatile damping coupling. The limited component technique is fit for giving all the essential data however its very tedious. Modeling programming can make the work truly less demanding and Ansys workbench is one of them.

\section{FINITE ELEMENT METHOD}

The Finite Element Method is a numerical method used for taking cares of engineering and mathematical problems. It is likewise alluded to as finite element investigation (FEA). Analytical method is 
exceptionally tedious however it gives a correct arrangement. Numerical methods are easier than analytical method and get approximate solution. Now a days scientists and engineers prefer numerical methods for their simplicity.

\section{METHODOLOGY}

\subsection{Design of Gear}

The gear was designed in solid works. It could be designed in Ansys but for designing of solid bodies solid works provides much easier interface than Ansys. Then the design was imported in Ansys. The parameters and geometry considered for the simulation is given in Table 1 and Figs.1-2.

Tab. 1. Geometry parameters of spur gear design

\begin{tabular}{ll}
\hline Number of teeth & $z_{1}=29$ and $z_{2}=31$ \\
\hline Face width & $12 \mathrm{~mm}$ \\
\hline Normal module & $3 \mathrm{~mm}$ \\
\hline Pressure angle & 20 degree \\
\hline $\begin{array}{l}\text { Range of rotating } \\
\text { speed }\end{array}$ & 10 to 10000 \\
\hline Torque range & 500 to $1500 \mathrm{Nm}$ \\
\hline
\end{tabular}

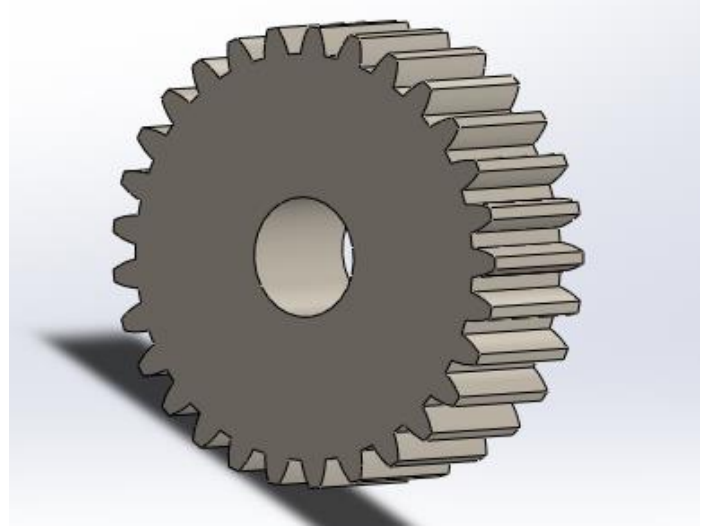

Fig. 1. Gear of 29 teeth

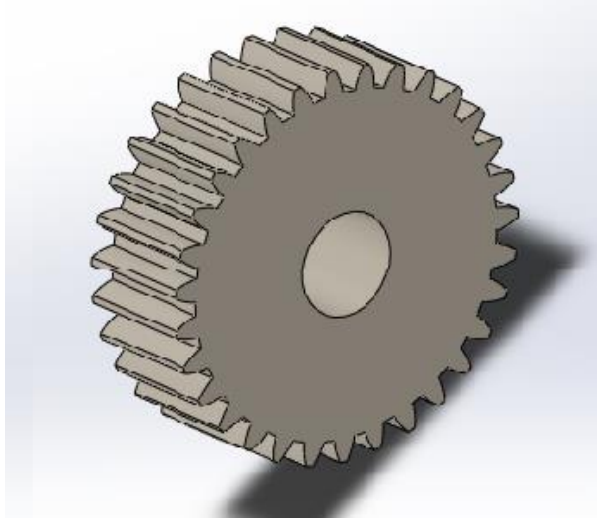

Fig. 2. Gear of 31 teeth

\subsection{Meshing and defining Boundary condition for stress analysis}

Element type solid10 node quadratic tetrahedral. Here both remote displacements for bearing supports are taken fixed (Tab. 2 and Fig. 3).

Tab. 2. Mesh sizing (Details of Mesh)

\begin{tabular}{ll}
\hline Relevance Center & Fine \\
\hline Initial size seed & Active assembly \\
\hline Smoothing & Medium \\
\hline Transition & Fast \\
\hline Minimum edge length & $1,47,480 \mathrm{~mm}$ \\
\hline
\end{tabular}

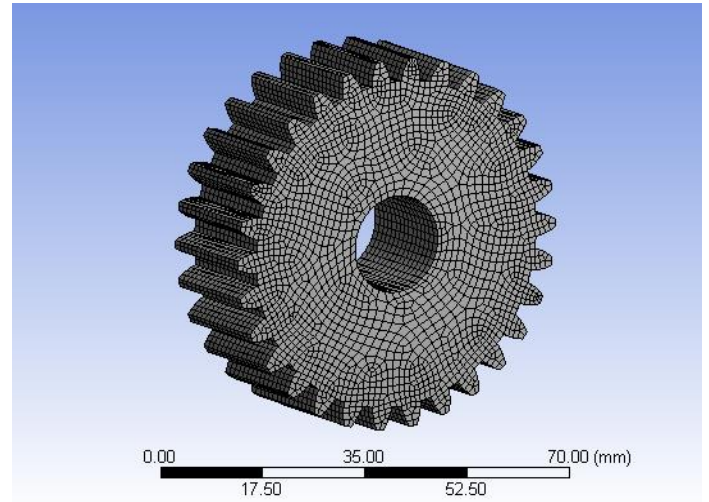

Fig. 3. Mesh of the gear 


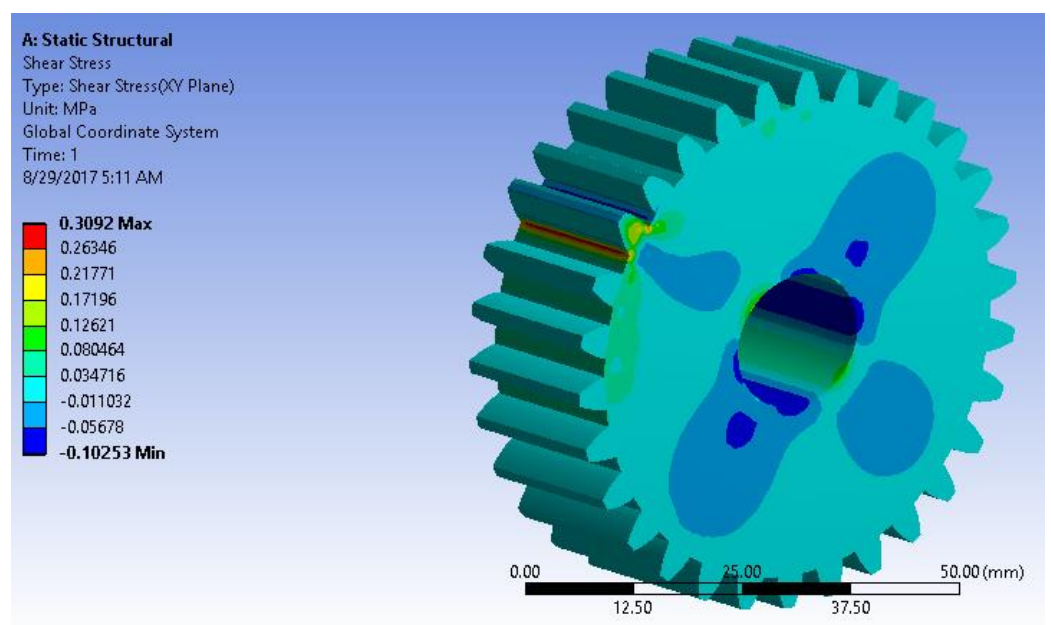

Fig. 4. Shear stress

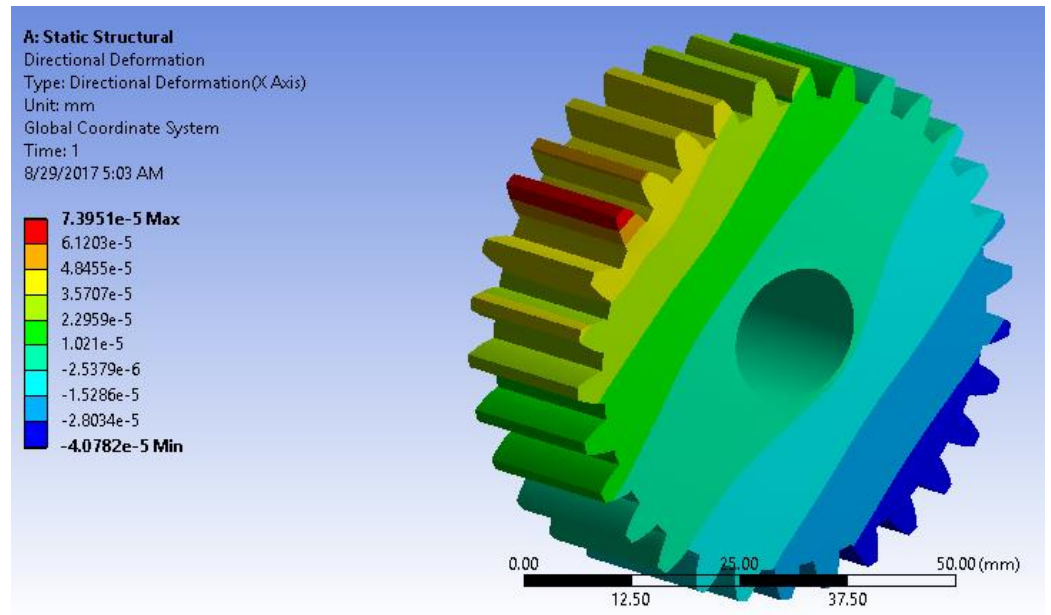

Fig. 5. Directional deformation

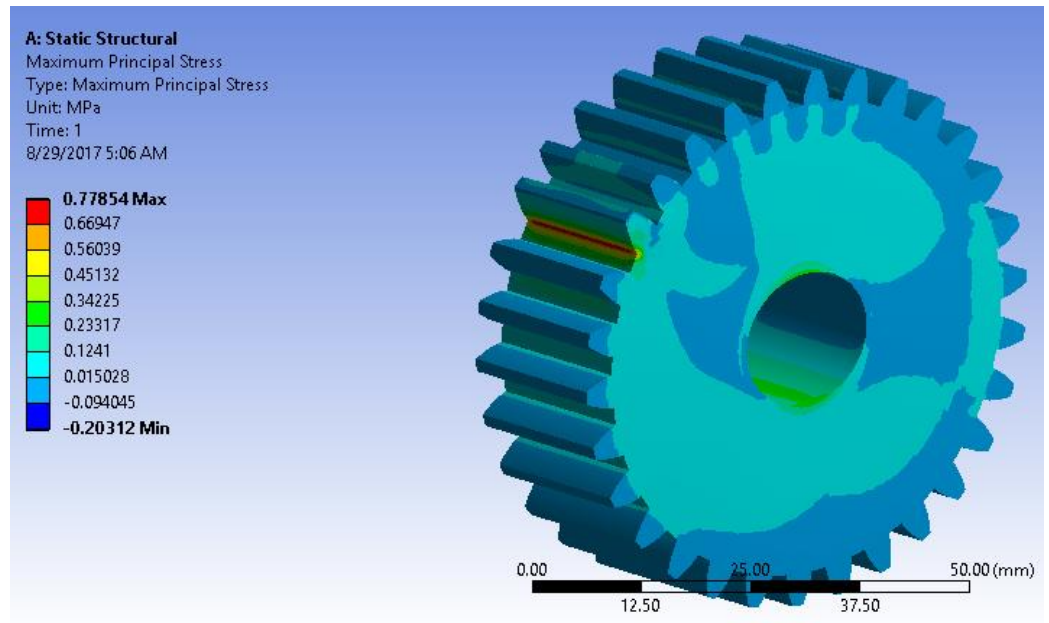

Fig. 6. Maximum principle stress 


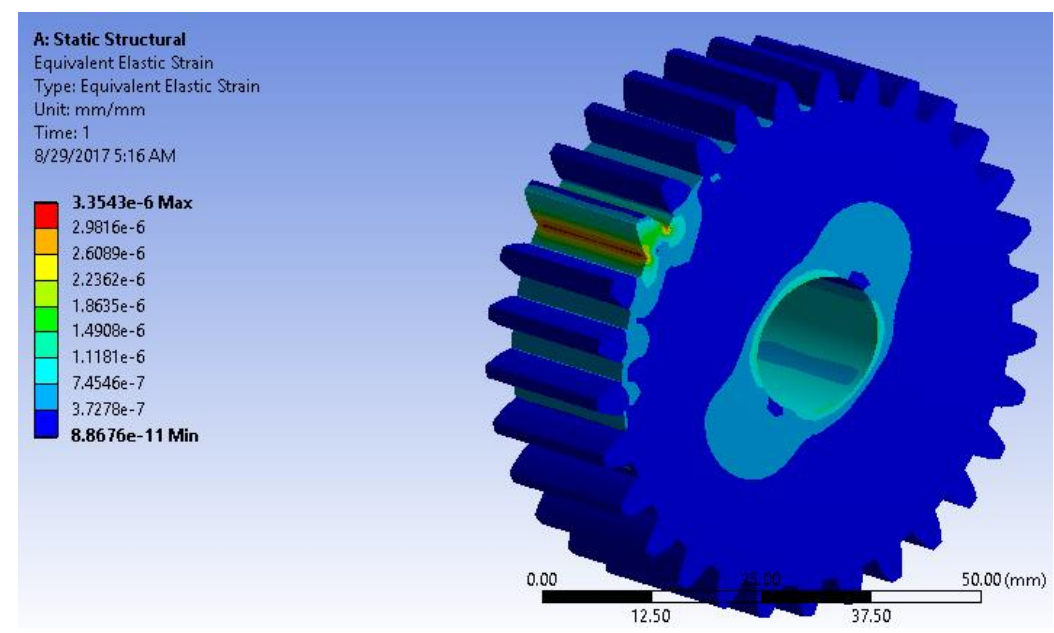

Fig. 7. Equivalent elastic strain simulation of gear system

The simulation was done by dynamic analysis. The numerical dynamic simulation using Ansys Transient Structural has been conducted through finite element method (Figs. 4-7). Figure 8 shows a bulk combination of two spur gears. The simulation results are presented in Figure 9 and Figure 10.

Figure 9 shows the dynamic bending stresses in the tooth at a speed of $50 \mathrm{rpm}$. This result is in good agreement with the experimental data of John Sinclaire Howland,1960 [9], which he investigated in 1960. Figure 10 demonstrates the simulation results about for the bending stresses in teeth roots. In this graph vividly identified point of resonance frequency as well as point of the increase of stress is half the speed of resonance.

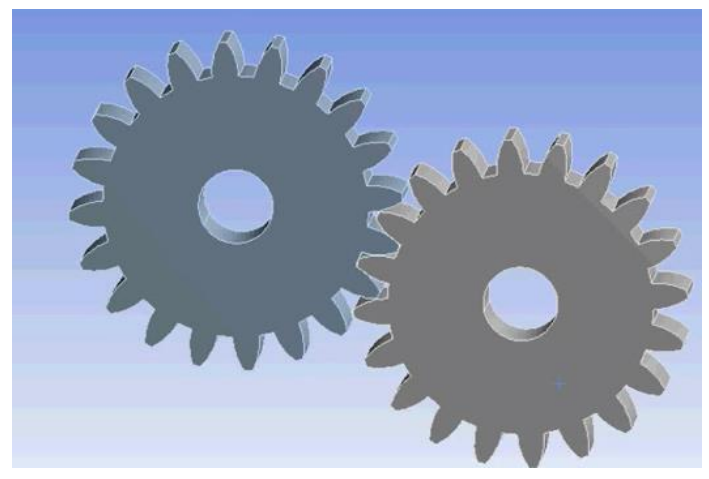

Fig. 8. Finite element method of spur gear pair in Ansys transient structural.

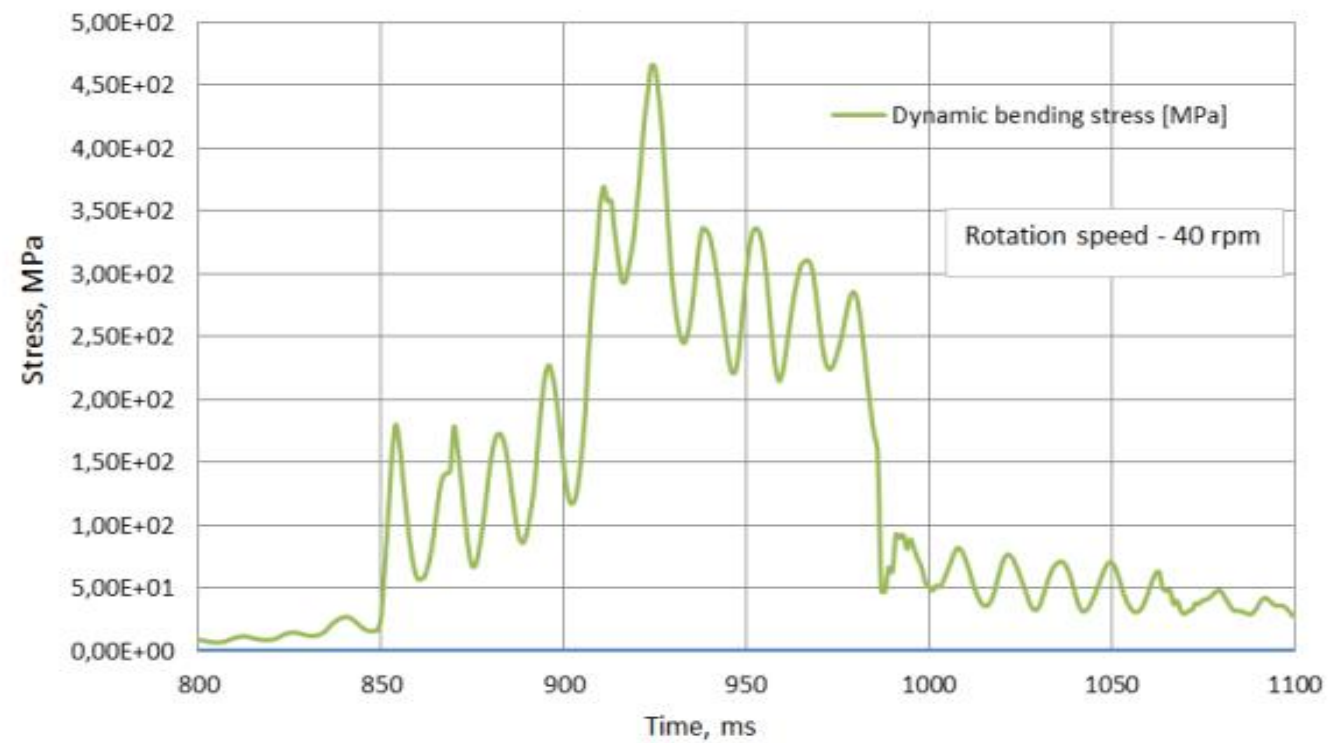

Fig. 9. Load distribution of bending stress for single tooth at a speed of $40 \mathrm{rpm}$ 


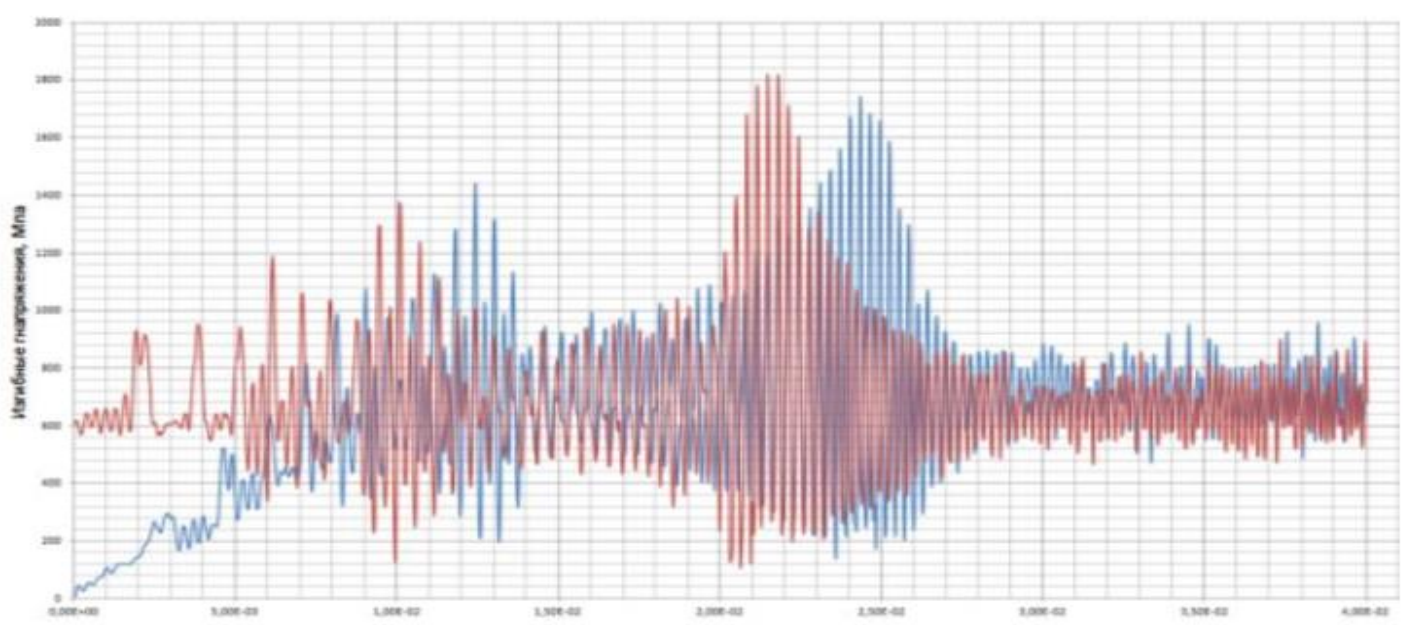

Fig. 10. Ansys transient simulation results: bending stress distribution with increasing rotation speed (blue) and reducing (red)

Figure 10 demonstrates the distinction between resonance frequency with the most extreme estimations of the bending stresses in the root when rotation speed is expanded (blue) and resonance frequency when rotation speed diminishes (red chart). Likewise demonstrated a noteworthy diminishment of dynamic stresses in supercritical range. This outcome might be utilized when planning the operation state of transmission.

\section{RESULTS AND DISCUSSION}

The finite element method is most broadly for locate a genuine model of the geared set utilizing the stress investigation in the combine of gears. The improvement of finite element investigation model of the spur gear assembly to reproduce the contact stress calculation and bending stress calculation is assume more critical part in the plan of gears. The investigation demonstrates that Hertz theory is the premise of contact stress calculation and Lewis equation is use for computing bending stress is a couple of gear. Theoretically result got by Lewis recipe and hertz equation and result found by tantamount with finite element investigation of spur gear. In The dynamic simulation results about demonstrated a decrease dynamic stress level in the gear prepare on basic range with increasing of external loads.

\section{CONCLUSIONS}

As a result, in view of this finding if the contact stress minimization is the essential concern and if the huge power is to be transmitted at that point spur gears with higher model is favored. Henceforth we reason that analysis software can be utilized for other dissecting reason. Results of demonstrating show affirmed the nearness of discontinuous vibration with loss of contact of the teeth in the basic range. Timeshifting mesh stiffness function inspects as principle wellspring of vibration and dynamic conduct of a gear framework and assess through nonlinear finite element model.

\section{Acknowledgements}

The authors are thankful to Dr. Jia-Chi Tsou, Professor, Dept. of Business Administration, China University of Technology, Taiwan for his undeniable support.

\section{References}

1. Arefin, M. A., Mallik, A., \& Asfaquzzaman, M. (2018). Renewable energy-assisted hybrid three-wheeler: A numerical investigation. Advances in Mechanical Engineering, 10(12), 1687814018814372.

2. Nasuti, F., Onofri, M., \& Martelli, E. (2005). Role of wall shape on the transition in axisymmetric dual-bell nozzles. Journal of propulsion and power, 21(2), 243250.

3. Jin, X., Wang, Y., Ju, W., He, J., \& Xie, S. (2018). Investigation into parameter influence of upstream deflector on vertical axis wind turbines output power via three-dimensional CFD simulation. Renewable Energy, 115, 41-53.

4. Liang, X., Zhang, H., Zuo, M. J., \& Qin, Y. (2018). Three new models for evaluation of standard involute spur gear mesh stiffness. Mechanical Systems and Signal Processing, 101, 424-434.

5. Mallik, A., Arefin, M. A., Rashid, F., \& Asfaquzzaman, M. (2017). Solar based plugged-in hybrid engine driven rickshaw (auto-rickshaw) \& its feasibility analysis for Bangladesh. In Proceedings of the international conference on mechanical, industrial and materials engineering RUET.

6. Mallik, A., \& Arefin, A. (2018). Micro Hybridized Autorickshaw for Bangladesh: A Solution to Green Energy Vehicle. The Open Mechanical Engineering Journal, 12(1)

7. Parker, R. G., Vijayakar, S. M., \& Imajo, T. (2000). Nonlinear dynamic response of a spur gear pair: modelling 
and experimental comparisons. Journal of Sound and vibration, 237(3), 435-455.

8. Karim, A. B., Avro, S. S., \& Shahriar, S. (2019). PROSPECT OF GEOTHERMAL ENERGY RESOURCES IN BANGLADESH. International Journal of Renewable Energy Resources, 8(2), 22-28.

9. Miller, J. M. (2006). Hybrid electric vehicle propulsion system architectures of the e-CVT type. IEEE Transactions on power Electronics, 21(3), 756-767.

10. Arefin, A., \& Islam, R. (2018). Investigation of Different Validation Parameters of Micro Gas Turbine for Range Extender Electric Truck. International Journal of Engineering, 31(10), 1782-1788.

\section{Biographical note}

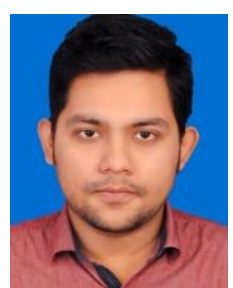

Anisul Islam is an undergraduate student of Dept. of Mechanical Engineering at Rajshahi University of Engineering \& Technology. His scientific interests focus on problems concerning machining processes and tools, efficiency, monitoring and diagnostics of machining processes as well as tribology. He has participated in 2 national research projects, presenting results of his work at a national conference. He has successfully completed his undergraduate thesis on 'Onion shell pyrolysis' and currently doing some independent researches. 